\title{
Las Administraciones públicas . y la promoción de la Sociedad de la Información: opciones estratégicas y modalidades de intervención
}

Jacint Jordana *

\section{La Sociedad de la Información y el papel de las Administraciones públicas}

Cada vez de forma más extendida, las Administraciones públicas se están enfrentando al reto de las transformaciones que las nuevas tecnologías de la información y la comunicación están produciendo actualmente en la economía y la sociedad. Tanto por cuestiones de eficacia y eficiencia, como por motivos de legitimación social, numerosas Administraciones públicas se encuentran en el proceso de incorporar en su relación con los ciudadanos, y también en todos sus procesos internos, las tecnologías y los procedimientos más convenientes para dar el mejor servicio posible y la más amplia y accesible información. Sin embargo, también es necesario reconocer que la difusión de las nuevas tecnologías de la información aún no se encuentra muy generalizada en las Administraciones públicas, a pesar de que ya existen numerosos ejemplos, que muestran las enormes posibilidades de relación directa con el ciudadano que ofrecen tales tecnologías.

Una utilización más intensiva de las tecnologías de la información por parte de la Administración pública es una tarea muy necesaria, sin duda, pero la cuestión que deseamos discutir es si las Administraciones públicas deben realizar, además, otras tareas relacionadas con la difusión de las nuevas tecnologías de la información y la comunicación. Queremos plantear la cuestión de que hasta qué punto fomentar la utilización de las mencionadas tecnologías, en todos los ámbitos sociales y económicos, es también una tarea prioritaria de las Administraciones públicas, o bien es una cuestión de carácter más secundario para el interés público.

En Europa, se ha proclamado, ya desde hace años, que incorporarse al desarrollo de la llamada Sociedad de la Información es claramente una de las grandes tareas de las Administraciones públicas contemporáneas (Informe Bangemann, 1994). El argumento central es que las nuevas tecnologías de la información están cambiando la forma de producir y de rela. cionarse, y se insiste en que los países y los territorios que incorporen de forma más rápida e intensa tales tecnologias, disfrutarán de mayores oportunidades de aprendizaje y de desarrollo económico y social en los próximos años. Evidentemente, no se trata de una relación totalmente automática, pero sin duda puede que exista una importante influencia ( $\mathrm{CAS}$. 
TELLS, 1997-1998). En todo caso, cada vez es más observable el impacto económico y social de lo que se está empezando a llamar la «nueva economía». En este sentido, puede que no sea suficiente que una Administración pública oriente sus preocupaciones en este ámbito sólo en el sentido de incorporar a sus procesos internos y a sus mecanismos de relación externa las tecnologias de información más actuales. Si el entorno con el cual establece su comunicación no incorpora tales tecnologías, o lo hace más lentamente, sus esfuerzos se verán escasamente recompensados; ya que de poco serviría de impulso al crecimiento económico una dinámica interna de las Administraciones públicas aislada del conjunto de la economía, más conectada con grandes organizaciones internacionales que con la realidad de su entorno territorial más próximo.

En este sentido, consideramos que promocionar activamente el desarrollo de la Sociedad de la Información en un entorno territorial concreto es, pues, uno de los retos primordiales de las Administraciones públicas en la actualidad, más allá de su propia incorporación como organizaciones que prestan servicios públicos. La utilización por parte de los usuarios particulares, la familiaridad con las nuevas tecnologías en los centros escolares y en los hospitales, la incorporación en las empresas de las nuevas prácticas comunicativas, etc., son elementos sobre los que las Administraciones públicas pueden incidir en alguna medida, al margen de las políticas de telecomunicaciones que cada gobierno emprenda para regular el mercado, privatizar los monopolios, o bien controlar los precios de las comunicaciones (KAHIN y WILSON, 1997; JORDANA, 1999).

\section{Las políticas públicas de telecomunicaciones y la promoción de la Sociedad de la Información}

A lo largo de los años noventa, en numerosos países, las políticas públicas de telecomunicaciones se han ido haciendo más complejas, definiéndose sofisticadas intervenciones regulativas que pretendían dinamizar el grado de competencia de sus economías. En esta década, muchos gobiernos han centrado sus esfuerzos en privatizar a los operadores de telecomunicaciones e introducir un mercado con mecanismos de libre competencia; o, por lo menos, separar la regulación pública de los aspectos propios de la gestión operativa de los servicios de telecomunicaciones (OECD, 1999; ITU,
1998). La gran transformación del sector de las telecomunicaciones producida en los últimos diez años, fruto de los cambios tecnológicos (digitalización, telefonía celular, etc.) y la fuerte tendencia a la globalización, con el objeto de alcanzar un mayor y más rápido desarrollo de las comunicaciones, han absorbido frecuentemente la atención de los gobiernos, que han tenido además que definir nuevos órganos de control y regulación, bien mediante la fórmula de las agencias autónomas, bien desarrollando nuevas áreas en sus estructuras orgánicas (MELODY, 1997). Una de las ideas centrales de toda esta transformación de las telecomunicaciones, inducida por los Estados, ha sido el convencimiento de que ello era un paso necesario para favorecer el desarrollo de la Sociedad de la Información, en el sentido de crear un nuevo marco que fomentara iniciativas empresariales más dinámicas en este sector.

Sin embargo, las dificultades afrontadas para construir un mercado de las telecomunicaciones mínimamente estructurado y competitivo han sido muy grandes en muchos países. Desde el mantenimiento de un predominio abrumador por parte del antiguo operador monopolista, hasta la entrada arrogante de los grandes operadores mundiales, pasando por las complejidades de construir un órgano regulador mínimamente capaz de imponerse frente a las empresas operadoras, o la feroz competencia entre grupos empresariales y financieros deseosos de obtener rápidas ganancias en un sector de fuerte crecimiento, los problemas experimentados por las políticas públicas de telecomunicaciones en esta década de grandes cambios han sido muy importantes, y no todos los países han conseguido encauzarlos con el mismo grado de éxito (ITU, 1995, 1998). Así, la fórmula generalmente utilizada, la privatización y el mercado, ha seguido procesos de aplicación muy distintos, más o menos rápidos, más o menos pautados con antelación, con mayor o menor grado de intervencionismo estatal, y también con mayores o menores resistencias internas (AHCIET, 1998). No es nuestra tarea entrar a analizar con detalle los distintos estilos de transición del monopolio al mercado experimentados en distintos países, y menos aún los resultados logrados en cada caso. Sin embargo, sí es necesario apuntar que los objetivos que se fijaron la mayor parte de los gobiernos al iniciar los procesos de privatización y liberalización, que consistían básicamente en aumentar el número de teléfonos por habitante, mejorar los estándares de calidad y en reducir las tarifas existentes, no se han cumplido en su totalidad, a pesar de que, sin duda, se han producido mejoras espectaculares en bastantes casos (OCDE, 1999; TAYLOR, WeBsTER y WILLLAM, 1996).

Estos temas, así como otros aspectos vinculados a la estructura de precios de las tarifas telefónicas y a la introducción de nuevas alternativas tecnológicas, continúan centrando en muchos casos las políticas nacionales de telecomunicaciones, 
una vez ya iniciada su liberalización y privatizados los antiguos monopolios. Ello ha contribuido a que el «instrumento» para estimular la Sociedad de la Información, se convirtiera en el centro de los debates políticos, sin que apareciera más intensamente la reflexión sobre la dirección que tomaba la propia Sociedad de la Información. Dado el elevado nivel de conflictividad política que ha generado en muchas ocasiones la liberalización de las telecomunicaciones, por los fuertes intereses económicos vinculados y por su gran impacto social, frecuentemente han quedado relegadas otro tipo de cuestiones, más relacionadas con la intervención pública en torno a los usos específicos de las redes y servicios de telecomunicaciones. Se trata de la discusión sobre cómo utilizar las políticas públicas para estimular determinadas modalida. des de uso productivo o creativo de las telecomunicaciones, con el apoyo de las nuevas tecnologías de la información, contribuyendo, desde el ámbito público, a definir el tipo de Sociedad de la Información del futuro o, en todo caso, a facilitar la difusión de una cultura de la información entre la población, que sirva de base -entre otros aspectos- al desarrollo de una economía de la información, que permita expandir la capacidad productiva de un determinado país (LUNDVALL y BORRÁs, 1998).

Por lo que se refiere a la promoción de la Sociedad de la Información desde el ámbito gubernamental, existen muchas posibilidades de actuación, y no hay duda de que algunos países ya han realizado una fuerte apuesta, empezando por el máximo nivel de liderazgo político, para apoyar e impulsar una difusión de la cultura de la información digital a todos los niveles de su sociedad, desarrollando políticas públicas específicas sobre este tema en múltiples ámbitos de la intervención pública (KAHIN y WILSON, 1997). Todo ello al margen, o por lo menos en paralelo, de las políticas específicas de liberalización del sector de las telecomunicaciones emprendidas por cada país. Sin embargo, en otros muchos casos las políticas de promoción, a pesar de algunos intentos, no han acabado de desarrollarse, en parte por los motivos destacados anteriormente, existiendo iniciativas interesantes, pero también muchas posibilidades de actuación sin desarrollar. Con el propósito de analizar las distintas estrategias posibles, y los problemas que limitan en cada caso su desarrollo, en este artículo se consideran las modalidades de intervención pública que se pueden utilizar para la promoción de la Sociedad de la Información, y se identifican distintos instrumentos de intervención utilizados, así como las posibilidades de planificación y coordinación existentes. Finalmente, se presentan distintos ejemplos de planificación e intervención para la promoción de la Sociedad de la Información, extraídos de algunas experiencias españolas y europeas.

\section{Fórmulas de la intervención pública para la promoción de la Sociedad de la Información}

Examinando los estilos de promoción existentes, podemos encontrar distintos puntos de vista sobre cómo promocionar la Sociedad de la Información y estimular directamente las nuevas tecnologías de la información por parte de la Administración pública. Un primer modelo conceptual considera que la cuestión clave es mejorar el funcionamiento del mercado, permitiendo la emergencia de todos los nuevos servicios y actividades que éstas pueden generar. Así, se supone que a medida que el mercado funcione mejor, la información circulará más rápidamente, redundando en una reducción de la incertidumbre que ayude a los actores empresariales a invertir en el sector y a desarrollar más rápidamente nuevas aplicaciones e innovaciones. Desde esta perspectiva, la Administración pública debe limitarse a facilitar la coordinación del mercado, a menudo mediante acciones simbólicas, o estructurando los grupos empresariales, y regular la competencia para evitar la reaparición de monopolios de facto. Entre los otros instrumentos utilizados, destacan los incentivos fiscales y la supresión de barreras comerciales (o creación de éstas, cuando convenga). Un segundo modelo conceptual parte del criterio de que la intervención pública, sea en forma de políticas activas que distribuyan bienes y servicios, sea creando ejemplos o impulsando la planificación, puede ayudar significativamente a impulsar la difusión y el uso de las nuevas tecnologías de la información en el mercado, acelerando su conocimiento y complementando las iniciativas privadas, cuando estas muestran dificultades en su desarrollo y crecimiento. El uso intensivo de instrumentos de planificación estratégica es un elemento básico de esta segunda concepción, ya que se considera muy importante la identificación y la coordinación entre actores públicos y privados, para que compartan, de forma explícita, los objetivos de desarrollo a alcanzar (SCHNEIDER, 1995).

Idealizando hasta cierto punto la realidad, podemos encontrar algunos rasgos destacados de estos dos modelos conceptuales en distintos ámbitos territoriales. En este sentido, el primer modelo se aproxima más a los principios norteamericanos de intervención, basados en defender la primicia del mercado por encima de cualquier intervención pública; mientras que el segundo modelo se encuentra más cercano a la orientación europea basada en la configuración de una esfera pública que tiene 
como reto el impulso global al desarrollo de la Sociedad de la Información, y donde participan de forma conjunta el sector público, las comunidades profesionales y las empresas privadas. Probablemente por este motivo, el término de Sociedad de la Información se ha acuñado y difundido en Europa, mientras que en los EEUU es mucho más común, al referirse al papel público la promoción de las tecnologías de la información y la comunicación, destacar el término - mucho más acotadodel desarrollo de las «infraestructuras de la información» ( $C_{A}$. TINAT, 1998). En este sentido, en las páginas siguientes nos centramos más en las distintas fórmulas de promoción que pueden encontrarse en el marco europeo, dejando al margen los mecanismos de promoción centrados exclusivamente en fortalecer los mercados.

Generalmente, las Administraciones públicas disponen de numerosos instrumentos de intervención para alcanzar los objetivos que se proponen, que aplican con intensidades y modulaciones distintas en numerosos campos de su entorno político, social y económico (ROSE, 1998). En la Tabla 1, se presenta una tipología muy simple de estos instrumentos de intervención, con una definición básica que describe la finalidad de cada tipo de instrumento. A partir de esta tipología, nuestro propósito es introducir con mayor claridad una discusión sobre las actividades más características que desarrollan las Administraciones públicas para fomentar la Sociedad de la Información. La tipología elaborada responde a una distribución de las prácticas de intervención presentes en las políticas públicas, discrimina. das según los efectos perseguidos y el grado de coacción sobre el comportamiento de los actores, que se pretende modificar en algún modo, mediante alteraciones de su estructura de incentivos y en el tipo de recursos que disponen.

\begin{tabular}{|l|l|}
\multicolumn{1}{|c|}{ Mabla 1} \\
Modalidades de intervención estatal \\
\hline \multicolumn{1}{|c|}{ Modalidad } & \multicolumn{1}{c|}{ Finalidad } \\
\hline Ejemplos & $\begin{array}{c}\text { Desarrollar una acción modelo con la finalidad de que sea imi- } \\
\text { tada desde el ámbito privado. }\end{array}$ \\
\hline Incentivos & $\begin{array}{c}\text { Establecer estúmulos con condiciones (premios) para modificar } \\
\text { el comportamiento de los ciudadanos. }\end{array}$ \\
\hline Oferta & $\begin{array}{c}\text { Establecer estímulos sin condiciones definidas sobre el com- } \\
\text { portamiento de los ciudadanos. }\end{array}$ \\
\hline Servicios & $\begin{array}{c}\text { Ofrecer a los ciudadanos bienes y servicios financiados y con- } \\
\text { trolados por entidades públicas (sin propósito de estímulo). }\end{array}$ \\
\hline Contratación & $\begin{array}{c}\text { Recibir bienes y servicios del sector privado para producir inter- } \\
\text { venciones públicas. }\end{array}$ \\
\hline Regulación & $\begin{array}{c}\text { Definir el comportamiento y las condiciones de interacción } \\
\text { entre los ciudadanos y las organizaciones públicas y privadas. }\end{array}$ \\
\hline Sanciones & $\begin{array}{c}\text { Establecer penalizaciones para los comportamientos no desea- } \\
\text { dos de los ciudadanos. }\end{array}$ \\
\hline
\end{tabular}

Fuente: Elaboración propia.
Así, nos encontramos desde las modalidades de intervención más suaves, como la utilización de ejemplos, con el propósito de generar procesos imitativos, hasta los procedimientos más intensos, como la aplicación de regulaciones o el establecimiento de sanciones que impliquen el ejercicio directo de la coacción estatal (WEINER y VinING, 1992; MÉNY y THOENIG, 1992). La utilización de páginas WEB para dar información detallada sobre la oferta de empleo público, facilitar el pago de impuestos a través de Internet, crear centros modelo que utilicen intensamente aplicaciones interactivas en ámbitos como la sanidad, la educación o el medio ambiente, son algunas iniciativas, que aparte de su valor intrínseco, tienen un efecto dinamizador muy importante, como muestra visible de nuevas actividades que pueden ser replicadas por numerosas iniciativas pertenecientes al ámbito privado. El empleo de incentivos en las políticas de promoción de la Sociedad de la Información es muy habitual, ya que éstos se orientan a modificar actitudes y comportamientos de la población. Así, desde ofrecer ventajas fiscales para el comercio electrónico, hasta los programas de apoyo financiero al desarrollo de iniciativas empresariales, pasando los estímulos a la investigación en temas clave del desarrollo de las nuevas tecnologías de la información, encontramos siempre en estos casos la existencia de una condición para recibir el apoyo público. La oferta se distingue de los incentivos por no existir un condicionamiento bien definido en el uso de los recursos públicos. Así, por ejemplo, la creación de centros públicos de libro acceso con infraestructura de acceso a Internet, o la distribución gratuita de aplicaciones interactivas son acciones típicas de oferta en este sector. La creación y distribución de nuevos servicios a traves de Internet es un tipo de actividad muy extendida entre las intervenciones públicas, a menudo mediante fórmulas de colaboración con el sector privado. El aceso a bases de datos públicas (registros, documentos, bibliotecas, etc.), el establecimiento de servicios de información (turística, empresarial, etc.), o poner a disposición de los ciudadanos espacios de debate y relación virtual, son algunas actividades frecuentes de este tipo.

Las otras modalidades de intervención que hemos destacado (contratación, regulación, sanciones), implican una utilización más destacada de la capacidad de coacción, sea en forma de amenaza, sea en forma de aplicación directa. En concreto, la contratación es un tipo de intervención que utiliza la propia capacidad de la Administración pública como organización para estimular procedimientos, introducir nuevas prácticas, obligar a los contratistas a seguir determinadas pautas, etc. Por ejemplo, introducir criterios tecnológicos en las bases de contratación pública, ofrecer toda la información sobre contratos públicos a través de Internet, son algunos de los mecanismos posibles. La regulación es una modalidad de intervención intensamente utilizada, acompañada frecuentemente de la capacidad de imponer sanciones. En este caso podemos encontrar líneas de actuación muy variadas, que van desde el desarrollo de un mar- 
co legal para el comercio electrónico, hasta la regulación del tratamiento de datos privados, pasando por la regulación de los propios dominios de Internet o la incoporación de requisitos de acceso a Internet para la construcción de nuevas edificaciones.

Junto a las modalidades de intervención estatal, también existe otra dimensión importante para analizar las políticas de promoción. Se trata del tipo de estrategia política y administrativa que predomina en cada caso. En este sentido, podemos identificar, entre las actuaciones de promoción desarrolladas en distintos países en los últimos años, cuatro tipos distintos de estrategias para impulsar las políticas de promoción de la Sociedad de Información (AlabaU, 1997; WILSON, 1997). En primer lugar, es posible observar Administraciones que no desarrollan un planteamiento estratégico global, sino que son sus distintas ramas sectoriales las que, en su caso, introducen elementos y planes de promoción específicos para impulsar las tecnologías de la información y la comunicación en su ámbito estricto, sea éste la educación, la sanidad o cualquier otro.

\begin{tabular}{|ccc|}
\hline \multicolumn{3}{c|}{ Tabla 2} \\
Opciones estratégicas en las políticas de promoción \\
de la Sociedad de la Información \\
\hline Opción estratégica & $\begin{array}{c}\text { Complejidad } \\
\text { administrativa }\end{array}$ & $\begin{array}{c}\text { Centralidad } \\
\text { política }\end{array}$ \\
\hline Planificación especializada sectorialmente & Media & Media \\
\hline $\begin{array}{c}\text { Promoción e impulso de las Administracio- } \\
\text { nes públicas }\end{array}$ & Baja & Media-Baja \\
\hline $\begin{array}{c}\text { Promoción e impulso del mercado y la socie. } \\
\text { dad civil }\end{array}$ & Media-Baja & Baja \\
\hline Planificación y coordinación intersectorial & Elevada & Elevada \\
\hline
\end{tabular}

Fuente: Elaboración propia, a partir de Alsau, 1997.

Una segunda opción consiste en concentrar la promoción e impulso de las nuevas tecnologías desde el sector público en definir nuevas estrategias de renovación y modernización de las propias Administraciones públicas. Desde esta perspectiva, se considera que el peso de la Administración pública como modelo y estímulo para el conjunto de la sociedad puede ser suficiente para conseguir los objetivos de la promoción propuestos. La tercera opción, la promoción y el impulso por parte del mercado y la sociedad civil, implica reconocer el papel subsidiario del sector público en este ámbito de políticas - acep. tando implícitamente los elevados riesgos existentes en su implementación-, y limitándose, por tanto, a ejercer de elemento coordinador de las iniciativas privadas, o a introducir recursos simbólicos en las actividades de promoción impulsadas por el sector privado - sea éste de carácter más social, o de carácter más empresarial-. Finalmente, una cuarta opción estratégica consiste en que la Administración pública impulse y coordine una planificación y una actividad de carácter plurisectorial, estimulando especialmente los aspectos más transversales. En este último caso, seguramente el más complejo y difícil de gestionar, se trata de aprovechar y potenciar las dinámicas cooperativas de carácter horizontal que permiten estas tecnologías, para impulsar cambios sociales y económicos que requieren complejos reajustes. En gran medida, resta implícito en esta última opción estratégica que los aspectos más sectoriales de las políticas de promoción de la Sociedad de la Información ya son incorporados en su propia planificación - de carácter rutinario- por las distintas Administraciones especializadas.

Como se muestra en la Tabla 2, estas distintas opciones estratégicas requieren, para su puesta en práctica con algunas garantías de éxito, un grado distinto de apoyo político. Así, la cuarta modalidad, basada en la coordinación intersectorial, tiene una necesidad imperiosa de recibir un apoyo constante desde las más altas instancias políticas del gobierno, para disponer de la capacidad de superar frecuentes tensiones entre sectores distintos de la Administración, y poder imponer y mantener objetivos comunes, cuando éstos sufren una tendencia a ser desvirtuados desde las distintas lógicas sectoriales. Por otra parte, la planificación sectorial, la primera estrategia, también requiere un cierto apoyo directo desde los niveles políticos más elevados, debido especialmente a que en numerosos sectores de actividad pública, la introducción de aplicaciones derivadas de las tecnologías de la información y la comunicación implica frecuentemente que se produzcan cambios en las distribuciones de recursos públicos y en las modalidades de intervención que existen en el sector, y ello puede generar inmovilismos o bien arreglos internos que den prioridad al statu quo existente, frente a las posibles mejoras existentes. Finalmente, los otras dos opciones estratégicas requieren una menor atención y apoyo político -especialmente la opción que da más protagonismo al sector privado-. Estas dos opciones no requieren un compromiso del conjunto de la Administración pública, o de grandes segmentos de ésta, sino que pueden ser más fácilmente impulsados desde unidades especializadas creadas para tal fin. Sin embargo, también es necesario reconocer que su efectividad para el propósito de impulsar la Sociedad de la Información depende en mucho mayor grado de cómo se comporten los contextos de la acción pública, y especialmente, de la presencia de una dinámica innovadora y cooperativa entre empresas y organizaciones de la sociedad civil.

Las distintas opciones estratégicas para el desarrollo de políticas de promoción de la Sociedad de la Información pueden generar distintos grados de complejidad administrativa en su puesta en práctica. Así, la planificación especializada sectorialmente (primera opción estratégica) seguramente concentrará 
sus esfuerzos en los procesos de diseño y planificación de las políticas, ya que las organizaciones concretas de ámbito sectorial que tomarán el protagonismo en esta dinámica generalmente están bien preparadas para estas tareas, mientras que tendrán mayores dificultades para asegurar una adecuada aplicación posterior, dado que sus unidades administrativas de carácter operativo pueden quedar más alejadas del entusiasmo innovador existente en la cúspide de la organización. La promoción basada en la renovación de la propia Administración (segunda opción estratégica) puede sufrir las dificultades habituales de las políticas de modernización de la Administración pública, que básicamente se limitan a desarrollar acciones de iniciativa y diseño, con graves dificultades para establecer luego una planificación detallada y las aplicaciones consecuentes. La opción basada en el protagonismo de la sociedad civil (tercera opción estratégica) tiene como acción más destacada la elaboración de iniciativas simbólicas y la creación de incentivos, que sirvan de referencia, impulso y coordinación a los actores privados. Finalmente, la opción de planificación intersectorial (cuarta opción estratégica) requiere dedicar una gran atención a las acciones de planificación, especialmente por lo que se refiere a la coordinación entre distintas organizaciones públicas y privadas, aunque sin descuidar los referentes colectivos que existen en el lanzamiento de una iniciativa común, así como el seguimiento centralizado de las aplicaciones sectoriales que se derivan de la planificación.

Para terminar este apartado, nos concentramos en la relación existente entre las distintas opciones estratégicas que pueden desarrollar las Administraciones públicas y sus gobiernos para impulsar la Sociedad de la Información, y las distintas modalidades de intervención estatal. En principio, cada una de las cuatro opciones estratégicas pueden incorporar en sus líneas de acción programas y actividades que tengan como referente cualquiera de las modalidades descritas en la Tabla 1, referidas a actividades de intervención pública para la promoción de la Sociedad de la Información. A pesar de ello, es evidente que, según la Administración y el nivel territorial que.concentre el peso de una determinada opción estratégica, las modalidades de intervención variarán, dependiendo de su capacidad normativa y su disponibilidad de recursos. También debe tenerse en cuenta que, independientemente de los recursos y las competencias, el tipo de opción estratégica determina una mayor intensidad en el uso de unas u otras modalidades de intervención. Así, la planificación sectorial seguramente hará un mayor uso de acciones basadas en incentivos, oferta y servicios, ya que busca un mayor contacto con los usuarios de sus actividades, mientras que la opción centrada en el impulso protagonizado por la propia Administración pública se concentrará en la contratación, los ejemplos y los servicios, ya que intenta ejercer de modelo que sea atractivo para imitar por parte del sector privado. La opción estratégica centrada en el impulso de la sociedad civil y el mercado debería centrar sus intervenciones básicamente en instrumentos de regulación y la con- tratación, dado que su objetivo es facilitar las iniciativas privadas. La última opción, la planificación y coordinación intersectorial, debería poder integrar un conjunto más variado de modalidades de intervención, ya que sus planteamientos son más sofisticados. Así, dado que el propio proceso de elaboración y aplicación de la política podría permitir detectar y corregir los efectos perversos que se producen, independientemente de los sectores para los que fueron pensadas las intervenciones, debería ser posible corregir y redefinir el conjunto de instrumentos utilizado cuando fuese necesario.

En ningún caso se pretende realizar en estas páginas un inventario de las intervenciones concretas que pueden llegar a realizarse. Evidentemente, como ya hemos señalado, todas las formas de intervención pueden presentarse mezcladas en la elaboración de programas públicos, y muchas de sus propiedades pueden ser idénticas a las que existan en otros ámbitos de políticas públicas. Sin embargo, una característica importante de este sector incipiente de políticas públicas es que, por su novedad, desconocemos en buena parte cuál es el grado de efectividad y estabilidad de cada instrumento, y su eficiencia en cada ámbito de actuación. Por ello, el grado de experimentación y de imaginación en las políticas públicas de promoción debe ser necesariamente muy elevado, lo que seguramente está provocando en la actualidad una gran variedad de resultados cuando se ponen en práctica estas políticas, algunos sin duda altamente positivos, otros con consecuencias más ambiguas. Sin embargo, a pesar de esta gran variabilidad, y de los elevados costes que implica la experimentación en la aplicación de políticas públicas, seguramente las políticas de promoción en el ámbito de la Sociedad de la Información sean extraordinariamente necesarias, si no deseamos vernos envueltos por muchos de los efectos perversos con que siempre nos envuelven las profecías sobre la Sociedad de la Información.

\section{Niveles de gobierno y prioridades de intervención}

Una de las primeras cuestiones relevantes al considerar las políticas de promoción de las tecnologías de la información y la comunicación es preguntarse cuál es el nivel de gobierno que puede ser más adecuado para llevar a cabo tales iniciativas. Por descontado que no encontraremos ninguna respuesta simple, ni en la realidad de los distintos países que más activamente han emprendido tareas de promoción, ni en un marco teórico que nos dé algún tipo de apoyo conceptual. 
Podemos encontrar actualmente ejemplos concretos de politicas de promoción sobre estos temas en todos los niveles de gobierno: desde organismos internacionales como el Banco Mundial o entidades supraestatales como la Unión Europea, pasando por múltiples iniciativas sectoriales de Administraciones de carácter estatal, regional o local. Al ser estas políticas de promoción bastante nuevas, en muchos casos no tienen una ubicación bien definida en la distribución de competencias entre los distintos niveles gubernamentales, surgiendo incluso en ocasiones de forma espontánea, sin una dirección política clara. En otros muchos casos, las políticas de promoción suelen partir de organizaciones públicas con una importante capacidad y visión estratégica, que están desarrollando algún tipo de misión vinculada a la promoción del territorio, actividades económicas o la atención al bienestar de sus habitantes. También es muy frecuente que se generen relaciones intergubernamentales entre los distintos niveles de Administración, como consecuencia de una emergente distribución de funciones en los procesos de definición e implementación de tales políticas de promoción.

En las políticas de promoción de la Sociedad de la Información podemos identificar con bastante frecuencia cuatro momentos distintos en su proceso de elaboración, aunque no tienen por qué estar todos presentes de forma independiente. En primer lugar, tenemos el origen de la iniciativa, el momento y el lugar en el que se concibe la idea, donde surge el impulso con los apoyos políticos y administrativos necesarios para que su impacto pueda generar posicionamientos públicos e iniciativas políticas concretas. En segundo lugar, podemos encontrar el momento del diseño de la política, cuando se definen los objetivos a alcanzar, los instrumentos de actuación, los recursos que se van a utilizar, etc. Estos dos momentos se encuentran muy relacionados, pero no tienen por qué estar siempre protagonizados por un mismo nivel de administración. En tercer lugar, podríamos situar el momento de la planificación. Se trataría aquí del proceso de priorización de los programas concretos de actuación, con la participación de los actores intervinientes, así como la sensibilización de estructuras administrativas sectoriales, etc. Existe sin duda también un cierto solapamiento entre la fase de diseño y la de planificación, ya que esta última puede integrar la primera, pero resulta interesante mantener la distinción, por la especificidad de estas políticas de promoción. Finalmente, en cuarto lugar, podemos encontrar el momento de la aplicación: cuando los programas se ponen en práctica, cuando las organizaciones públicas o privadas actúan territorial y sectorialmente aplicando instrumentos de intervención específicos, que inciden sobre la realidad económica y social de su entorno.

Seguramente, para otras políticas más establecidas, que no se encuentran en un momento emergente, no sería necesario identificar tan claramente algunas de estas fases, lo que nos llevaría a utilizar modelos más clásicos sobre las fases de ela- boración de las políticas públicas. Por ello, consideramos que se trata, por tanto, de una especificidad de este tipo de políticas vinculadas al desarrollo de las tecnologías de la información y la comunicación, ligada a su novedad, y provocada por la discusión e incertidumbre existente, tanto sobre su necesidad como sobre su efectividad. Por estos motivos, es frecuente que los momentos de su diseño y formulación se dilaten enormemente en el tiempo, mientras que los momentos de su implementación sean mucho más concretos y puntuales. Ello conduce a que el debate y la reflexión sobre la capacidad y la justificación para la intervención pública en este sector, o sobre los instrumentos de actuación más adecuados, centren frecuentemente buena parte de los esfuerzos de las propias Administraciones públicas en este ámbito.

A modo de ensayo, y partiendo de numerosas observaciones de iniciativas y políticas desarrolladas en los últimos años (recogidas, por ejemplo, en ISPO), en la Tabla 3 se realiza una propuesta que estructura algunas de las distribuciones más probables de funciones entre distintos niveles gubernamentales, que pueden llegar a definir distintos modelos de relaciones interadministrativas. Así, podemos encontrar procesos de elaboración e implementación de políticas de promoción que se inician en niveles de gobierno supranacionales, y encuentran finalmente su implementación en el ámbito local o regional, hasta situaciones en las que los procesos se inician y se concretan com. pletamente en un mismo nivel.

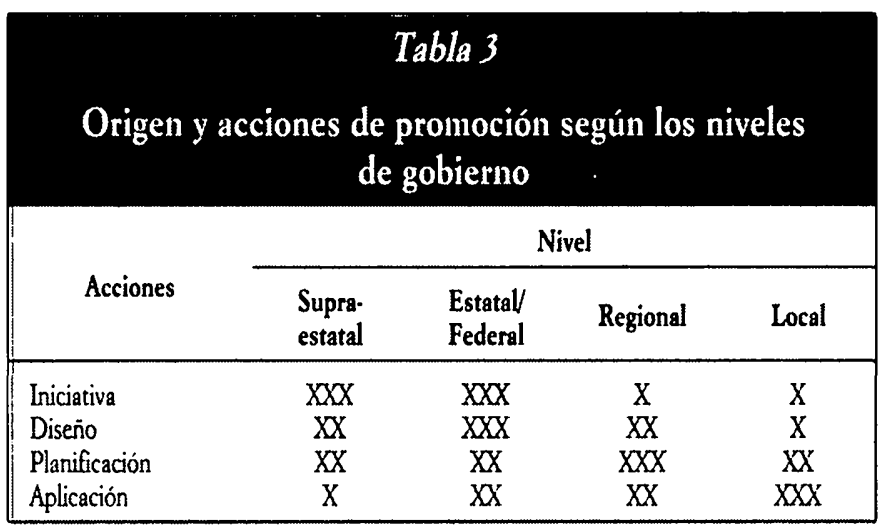

$\mathrm{XXX}$ : Muy frecuente; XX: Frecuente; X: Poco frecuente.

Fuente: Elaboración propia.

Si localizamos en cada caso dónde se sitúa la iniciativa y el diseño de las políticas, frecuentemente es posible identificar cuáles son los actores públicos con mayor capacidad de impulso y anticipación, frente a otros actores también implicados en estas políticas, pero con un comportamiento más reactivo. Un aspecto a destacar en estas políticas es que las Administraciones más activas no tienen por qué encontrarse siempre situadas en los niveles más elevados de gobierno. Pueden existir incluso casos de Administraciones locales que autónomamente deciden 
impulsar y diseñar programas de promoción en su ámbito territorial. Estas iniciativas incluso pueden llegar a generar unos procesos de imitación muy intensos, actuando como ejemplos para otras Administraciones locales o regionales. También hay que tener presente que se pueden producir solapamientos, sin una coordinación explícita, entre las acciones de promoción que son impulsadas por distintos niveles de gobierno, y que se aplican sobre un mismo entorno territorial.

En cualquier caso, los objetivos de las políticas de promoción intentan conseguir la máxima efectividad y difusión territorial mediante estímulos bien seleccionados, dados los limitados recursos que disponen. Para ello, las lógicas de intervención difieren según el nivel administrativo que tenga el protagonismo del impulso. Así, en el caso de las iniciativas llevadas a cabo desde entidades supranacionales, los planteamientos se orientan generalmente a sensibilizar los otros niveles de gobierno, fundamentalmente los estatales, sobre la necesidad de establecer planes y programas de promoción y desarrollo de la Sociedad de la Información. Tampoco es completamente extraño que en ocasiones exista una relación directa entre el nivel supraes. tatal y el nivel regional, ya que las organizaciones supraestatales buscan implicar Administraciones experimentadas, con capacidad efectiva de actuación sobre el territorio, y, por lo tanto, si el entorno político-institucional lo permite, este tipo de combinaciones puede articularse rápidamente, de forma bastante eficiente.

En numerosos países - seguramente de forma mayoritariahan sido las Administraciones federales o estatales las que han tomado la iniciativa y han definido un marco global de promoción, impulsando y definiendo programas específicos de actuación y estableciendo mecanismos de coordinación intersectorial. La gestión de tales programas ha seguido procedimientos muy variados, dependiendo de la articulación política de cada país y de sus tradiciones de descentralización. Posiblemente, la estructura institucional y la articulación de intereses presente en cada país hayan sido elementos clave para influir en el grado de control de los recursos destinados a la promoción por parte de las Administraciones públicas regionales y locales. Si los núcleos de actores más influyentes en los temas relacionados con las nuevas tecnologías de la información y la comunicación se encuentran muy cohesionados alrededor de las instituciones estatales o federales, entonces la capacidad de las Administraciones de los niveles inferiores para desarrollar iniciativas propias probablemente será bastante más limitada. Asimismo, si las Administraciones más centrales disponen de una fuerte capacidad de coordinación intersectorial y ejercen un fuerte impulso político, también se reducirá el abanico de oportunidades para la libre iniciativa de las Administraciones locales y regionales en este ámbito.

\section{Algunos ejemplos de iniciativas de promoción de la Sociedad de la Información}

Este apartado está dedicado a presentar algunos ejemplos de iniciativas de promoción de la Sociedad de la Información (referentes al período 1996-1999), con el objeto de ilustrar las distintas modalidades de intervención pública discutidas en las páginas anteriores. No se trata de realizar un recuento sistemático de casos, ni de analizar con profundidad distintas experiencias concretas. Sencillamente, se trata de ubicar, a partir de casos reales, algunas de las posibles situaciones planteadas de forma conceptual en los apartados previos. Se destacan tres casos originados en distintos niveles territoriales: una iniciativa promovida por la Unión Europea, una propuesta de actuaciones sobre un entorno local y un programa estatal llevado a cabo por dos organismos ministeriales de forma conjunta, ambos referentes al territorio español.

\section{La iniciativa europea RISI (Regional Information Society Initiative)}

La Comisión Europea impulsó en los años noventa, básicamente desde la Dirección General XIII, diversos programas de promoción y difusión de la Sociedad de la Información. Por una parte, se aplicaron abundantes fondos estructurales al desarrollo de las infraestructuras de telecomunicaciones en las áreas territoriales menos desarrolladas (LANDÁBURU, 1997). Por otra parte, desde otros ámbitos comunitarios se seguía impulsando la investigación en este campo, frecuentemente en coordinación con empresas europeas del sector, y se apostaba por la planificación a largo plazo de los desarrollos tecnológicos y por la fijación consensual de nuevos estándares para las futuras generaciones de instrumentos, redes y equipos de telecomunicaciones (Hedblom y GarRISON, 1997).

Una de las iniciativas complementarias a estos programas e intervenciones fue la propuesta RISI, que tenía como objeto la integración del concepto de Sociedad de la Información en las políticas de desarrollo regional. En una primera fase experimental se escogieron seis regiones europeas, y, posteriormente, en una segunda fase, se generalizó la iniciativa a un conjunto de 28 nuevas regiones, incorporando territorios de todos los países miembros de la Unión Europea. El contenido de esta 
iniciativa consistía en dar los apoyos necesarios para sensibilizar a los actores regionales clave de cada territorio sobre la necesidad de establecer un consenso y cooperar para promover la Sociedad de la Información. Así, para conseguir esta finalidad, se impulsaba y se diseñaba una metodología para la elaboración de planes estratégicos a escala regional que, en cada caso, debían ajustarse a las posibilidades y necesidades de cada región.

Los resultados obtenidos en cada región en términos de promoción del consenso, así como la continuidad en el desarrollo de aplicaciones concretas, son aspectos que requerirían un análisis específico en cada caso, pero lo más destacable de esta iniciativa en su conjunto son sus características conceptuales: se trata de una política sin aplicaciones concretas, ya que sólo contempla acciones de iniciativa, diseño y planificación, relacionando dos niveles de gobierno no continuos (supraestatal y regional), y que combina dos modalidades de intervención muy distintas: la creación de ejemplos (sólo se seleccionaron un pequeño número del total de regiones europeas existentes) y la provisión de incentivos (se ofrecieron recursos específicos para la coordinación, la preparación de la planificación y para facilitar la participación de los actores clave regionales). Por otra parte, la iniciativa no proponía de forma exclusiva una opción estratégica concreta. Aunque podía sugerir mecanismos de planificación intersectorial, finalmente quedaba en manos de cada región la elección de su apuesta estratégica, especialmente por lo que se refiere a la concreción de las medidas concretas a aplicar.

\section{Un caso de promoción local de la Sociedad de la Información: Mataró}

Esta iniciativa se localiza en una población de unos 100.000 habitantes, próxima a la ciudad de Barcelona, con una actividad industrial centrada en el sector textil y una fuerte presencia de sectores comerciales y de servicios. A partir de inicios del año 1998, distintos actores locales empiezan a impulsar la idea de realizar un plan director para el desarrollo de las nuevas tecnologías de la información y la comunicación en el ámbito local, iniciativa que es asumida por el ayuntamiento de la ciudad, dándole el apoyo necesario para su realización. Su fase de diseño se extiendió durante bastantes meses, e incluyó la realización de encuestas específicas a los ciudadanos, así como una muestra específica dirigida a empresarios, con el objeto de realizar un diagnóstico sobre la utilización de las tecnologías de la información y la comunicación en el ámbito local. Paralelamente, realizaron un inventario de experiencias locales y foráneas que pudieran servir de inspiración para las propuestas a realizar. En otoño del mismo año, se inició el momento de elaboración de la planificación propiamente dicha, mediante un procedimiento ampliamente participativo en el ámbito local, basado en la creación de diversos grupos de trabajo.

Los grupos de trabajo, con la participación de numerosos representantes de empresas, sector público y de todo tipo de asociaciones locales, se articularon en dos ejes distintos: la implantación de las tecnologías de la información y la comunicación (infraestructuras, servicios, usuarios), y el desarrollo de la ciudad (social, económico y de la propia Administración). Finalmente, se constituyeron siete grupos distintos, todos ellos incorporando una perspectiva horizontal que debía interrelacionar elementos de los dos ejes mencionados. Cada grupo de trabajo debía elaborar un conjunto de propuestas de directrices, objetivos y acciones concretas, que finalmente deberían ser revisadas por los órganos de coordinación y dirección del plan. Todas las propuestas debían tener como referente la necesidad de posicionar adecuadamente a la ciudad frente al reto de la Sociedad de la Información, destacando especialmente cuál debía ser el posicionamiento del sector público local en esta dinámica.

En su conjunto, y hasta finales de 1999, la actividad desarrollada con relación a la promoción de la Sociedad de la Información se centró en impulsar la iniciativa y desarrollar las tareas de planificación, mediante un procedimiento ampliamente participativo. Se iniciaron ya algunas aplicaciones, pero éstas no se derivaban directamente del plan, aunque posiblemente surgieron en el contexto dinamizador que se desarrolló con la elaboración del plan. Aunque el plan tenía unas pretensiones de intervención multisectorial, lo que presuponía una intensa coordinación interna, la realidad del sector público local conllevó que finalmente éste se inclinara más hacia algunos ámbitos concretos vinculados a sus propias capacidades de intervención, como fueron la educación, la promoción de contenidos y el estímulo a las infraestructuras (TEODORO y ARDERIU, 1999).

La utilización de servios públicos, ejemplos e incentivos concentraba buena parte de las actuaciones propuestas. Estos aspectos mencionados situarían el plan en la dirección de la opción estratégica intersectorial, aunque hay que mencionar también la presencia de una dimensión adicional, centrada en reforzar la capacidad de impulsar la coordinación entre el propio sector privado empresarial, dinámica que los objetivos del plan no descuidaban en ningún modo.

\section{Programas de promoción de la Sociedad de la Información de un departamento ministerial en España}

A principios de los años ochenta no existía regulación ni gestión pública en este ámbito en España, más allá de lo que la propia Telefónica, como empresa, pudiera desarrollar. La primera Ley de Ordenación de las Telecomunicaciones fue 
promulgada en 1987, y la creación de la Dirección General de Telecomunicaciones se produjo en el año 1985 y, a partir de entonces, la intervención pública, separada de la actividad de Telefónica, fue creciendo, pero lenta y tímidamente, especialmente en los primeros años, definiendo progresivamente sus propios objetivos (NADAL, 1995). Por este motivo, en aquellos años la capacidad de la Administración pública para utilizar Telefónica como implementadora de sus políticas era realmente muy escasa, y sucedía más bien al contrario, ya que Telefónica, como organización, articulaba su propia estrategia de desarrollo, incluyendo también, evidentemente, consideraciones de interés público (MarTínEZ, 1998; JoRDANA, 1999).

A lo largo de los noventa, al prepararse para la competencia y la privatización, Telefónica fue dejando cada vez más de lado este tipo de políticas, despreocupándose hasta cierto punto de estas cuestiones, tanto financiera como estratégicamente. La internacionalización de la empresa era, por el contrario, la nueva prioridad, compartida incluso por el propio gobierno. Ya con la privatización, en 1997, la desvinculación de Telefónica de los objetivos de políticas públicas en el ámbito de la promoción fue casi total, como lo evidenció el desmantelamiento de Fundesco, la fundación que Telefónica utilizaba para gestionar muchas actividades de promoción y difusión, frecuentemente en conexión con programas europeos.

En este contexto, puede decirse que -por lo menos hasta 1999- la labor de impulso y promoción de las telecomunicaciones que realizaba Telefónica apenas fue recogida por la Administración, dejando aparte las actividades de la Secretaría General de Comunicaciones (SGC), perteneciente al Ministerio de Fomento, el organismo que tenía las competencias de regulación y control de las telecomunicaciones. Sin embargo, no se establecieron nuevas partidas presupuestarias encaminadas a sustituir las tareas que hasta entonces estaba realizando Telefónica. En todo caso, muchas de las actividades que se fueron realizando a partir de entonces por el sector público fueron producto de algunos nuevos desarrollos y programas impulsados por la Comisión Europea.

Una excepción importante a la situación descrita fueron las actividades de promoción de la Sociedad de la Información impulsadas desde la SGC, entre las que cabe destacar el programa PISTA (Promoción e Identificación de Servicios Emergentes de Telecomunicaciones Avanzadas), con diversas líneas de acción definidas en distintos ámbitos de intervención. En este contexto, una de las líneas de acción que tomó más protagonismo fue el proyecto PISTA-Ventanilla Única, realizado en coordinación con el Ministerio de Administraciones Públicas, y que tenía el propósito de desarrollar un sistema intercomunicado de registros en el que participaran las distintas Administraciones públicas existentes en España (estatal, autonómica, local), con el objeto de simplificar y facilitar la comunicación entre los ciudadanos y la Administración con la ayuda de ins- trumentos telemáticos, tanto para tramitaciones, como para la información y comunicación (NISTAL, 1999).

Este proyecto constituía un elemento más de la estrategia de modernización de las Administraciones públicas, y, en este sentido, implicaba una opción estratégica basada en la promoción e impulso a partir de las Administraciones públicas, mediante una modalidad de intervención basada en el desarrollo de servicios (y algunos elementos de regulación). Sin embargo, hasta cierto punto, el conjunto de la aplicación también contenía el propósito de que sirviera de modelo para el sector privado, como referente de aplicación general. En cuanto al ámbito territorial, esta acción de promoción se impulsó, se planificó y se desarrolló en el nivel estatal, sin vincularse de forma estable a otros niveles administrativos; aunque estaba pensado que su implantación y aplicación se pudiera realizar en todos los niveles de la Administración pública - y en este sentido si existía una relación operativa entre niveles de gobierno-. Sin embargo, dada la complejidad de los niveles de Administración pública en España, y la frecuente necesidad de establecer mecanismos de cooperación y consenso interadministrativo, este programa afrontó el riesgo de llegar a estar demasiado vinculado a la Administración promotora, con lo que se podían generar algunas dificultades —de carácter político y organizativo- para su aceptación por el resto de niveles de gobierno.

\section{Conclusiones: el predominio del debate público y centralidad de la política}

A lo largo de las páginas anteriores, se ha analizado un conjunto de variables clave para identificar y discriminar las intervenciones públicas que tienen como propósito la promoción de la llamada Sociedad de la Información. La intención explícita era establecer una cierta guía, con el objeto de ofrecer una orientación sobre el entorno actual en este sector de políticas, cada vez más poblado de iniciativas de todo tipo. Por el momento, difícilmente puede discutirse en términos generales la efectividad de estos programas e intervenciones, ya que existe muy poca experiencia, y, especialmente, porque -como hemos visto- es muy frecuente en este ámbito de políticas que no se generen de forma inmediata intervenciones efectivas sobre la sociedad y la economía, sino más bien que se produzcan frecuentes procesos de discusión pública y largos procedimientos para la elaboración de planes y programas — cuestiones ambas, 
que en sí mismas no dejan de constituir ya una clara contribución a la dinamización de la Sociedad de la Información-.

Por otra parte, la dinámica de cambio acelerado que se está produciendo aún complica mucho más la lógica de la intervención pública, especialmente por lo que se refiere a la definición de los objetivos de la intervención. Los parámetros del contexto sobre el que puede actuar el sector público se modifican tan rápidamente (crecimiento exponencial de los usuarios de Internet, oscilaciones imprevisibles de las opciones tecnológicas, transformaciones de la estructura de los mercados, evolución de la convergencia digital, etc.) y, además, con tal incertidumbre, que frecuentemente sobrepasan su propia capacidad de reacción. Así, la interrelación entre la especial situación de desarrollo acelerado en que se encontraba este sector y la especificidad emergente de las políticas públicas en este sector —con la lentitud ya reseñada - puede conducir fácilmente a retrasos en las respuestas públicas, o situaciones relativamente paralizantes, en las que sea especialmente complicado mantener objetivos públicos coherentes, así como conseguir realizar intervenciones efectivas en los momentos más adecuados. No es extraño que cuando se logra articular una actuación pública, el tiempo que se consume a continuación para definirla y planificarla, obligue a volver a revisar los criterios iniciales con los que se impulsó la actuación, llegando incluso a frenar la aplicación efectiva de la intervención. En resumen; la tensión para la intervención pública consiste en que los riesgos de las acciones públicas en este ámbito a menudo parecen demasido elevados, a la vista de cómo evoluciona el sector, pero, por otra parte, los riesgos de la parálisis o la inacción sobre estos temas también pueden ser iguales o más elevados.

A pesar de las mencionadas dificultades, existen algunos puntos destacados que, de una forma más implícita, pueden deducirse del análisis realizado hasta el momento sobre la lógica de las políticas de promoción de la Sociedad de la Información. Reconocerlos puede contribuir a un cierto aprendizaje en el proceso de elaboración de estas políticas y ayudar a superar en lo posible los efectos paralizantes que hemos destacado. Concluimos, por tanto, identificando cinco problemas clave para las políticas de promoción de la Sociedad de la Información.
En primer lugar, parece bastante claro que, en las condiciones actuales, cuanto más ambiciosos y extensos sean los objetivos de la promoción, más difícil e improbable es que los planes y programas alcancen una fase de aplicación operativa, siendo más probable que se queden estancados en el momento del diseño y la planificación. En segundo lugar, cabe destacar que uno de los obstáculos más graves para la promoción de la Sociedad de la Información es la dificultad de implicar efectivamente a las Administraciones especializadas sectorialmente, ya que éstas pueden entender que tales políticas suponen cargas adicionales. Por ello, el problema es evitar que los objetivos de la promoción sean asumidos exclusivamente por un núcleo especializado dentro de la Administración. Un tercer elemento importante es valorar el largo proceso de elaboración de estas políticas de promoción. Aunque pueda parecer algo ineficiente, tiene el efecto positivo de crear un nuevo escenario público, que sirve para facilitar la coordinación entre actores públicos y privados, sensibilizarlos sobre estos temas, y también fomentar su participación en la definición de objetivos. La implicación ciudadana debería constituir, a la vez, un objetivo de estos programas de promoción y un requisito en su elaboración y puesta en práctica. Conseguir combinar ambos propósitos puede ser un factor clave de éxito en la difusión de una cultura avanzada sobre las tecnologías de la información y la comunicación. En cuarto lugar, es necesario reconocer que aún existe un elevado desconocimiento sobre la eficacia de muchas de las intervenciones posibles, y que, por lo tanto, especialmente respecto a las acciones más directas, el grado de experimentación e incertidumbre en este tipo de políticas es muy grande.

Finalmente, un quinto elemento a tener muy presente en las políticas para la promoción de la Sociedad de la Información se refiere al papel de los apoyos políticos. La existencia de un fuerte liderazgo con criterios políticos claros sobre la importancia de este tema, así como el apoyo constante a las políticas de carácter más horizontal, que requieren un elevado grado de coordinación, constituyen aspectos cruciales para el éxito de las políticas de promoción, cuyo influjo debería alcanzar más allá de los primeros momentos de éstas, superando los momen. tos iniciales de impulso y definición. 
ALABau, A. (1997), «Telecommunications and the Information Society in European regions», Telecommunications Policy, $21,8$.

Castells, M. (1997-1998), La era de la información: economia, sociedad y cultura, 3 vols., Madrid: Alianza.

Catinat, M. (1998), «La politique américaine d'Infraestructures d'Information», Communications and Strategies, 29.

Hedblom, Milda, y GarrISON, William (1997), «European Information Infraestructure Policy Making in the Context of the Policy Capacity of the European Union and Its Member-States: Progress and Obstacles», en B. KAHIN y E. WILSON, National Information Infraestructure Initiatives, op. cit.

Informe Bangemann - Unión Europea (1994), Europa y la Sociedad global de la Información, Bruselas.

International Telecommunication Union (1995), World Telecommunications Development Report. Universal Access, Ginebra.

International Telecommunication Union (1995), World Telecommunications Development Report. Information Infraestructures, Ginebra.

JoRDANA, J. (1999), «El camino hacia la sociedad de la información. ¿Son útiles los planes y las políticas para su promoción?», en J. Jordana y D. SanCho (1999), Politicas públicas de telecomunicaciones en España, Madrid: Tecnos.

KaHIN, B., y WusON, E. (1997), National Information Infraestructure Initiatives, Cambridge: MIT Press.

LANDÁBURU, Eneko (1997), «Desarrollo y cohesión social: las infraestructuras como elemento indispensable para el desarrollo regional. Los fondos estructurales», Telecomunicaciones 1996. Tendencias, Madrid: Fundesco.

LUNDVAL, B. A., y BORRAS, S. (1998), The globalising leaming economy: implications for innovation policy, Comisión Europea, DGXII.

MARTinez Soler, José A. (1998), «Telecomunicaciones: un sector en ebullición», Economistas, núm. 77.
Melody, W. (ed.) (1997), Telecom Reform. Principles, Policies and regulatory Practices, Lynby: Technical University of Denmark.

MénY, I., y ThoenıG, J. C. (1992), Las politicas públicas, Barcelona: Ariel.

NADAL, Javier (1995), «La fusión de las telecomunicaciones y el multimedia. Perspectiva española», Estudios de Transportes y Comunicaciones, núm. 66.

NisTAL, G. (1999), «El proyecto Ventanilla Única. Construyendo la Sociedad de la Comunicación», III Jomadas de Organización y Nuevas Tecnologías en la Administración Local, Blanes, 6-8 de octubre de 1999.

OECE (1999), Communications Outlook 1999, Paris.

ROSE, R. (1998), El gran gobiemo. Un acercamiento desde los programas gubermamentales, México: FCE.

SCHnemer, V. (1995), «Different roads to the Information Society? Comparing the US and the European approaches from a comparative public policy perspective», paper presented at the International Conference «The Social Shaping of IS -Comparing the NI and the EU Action Plan», Bremen, 5.7 de octubre de 1995.

TAYLOR, J. A.; WEBSTER, C., y WlLLAM, R. (1996), «Universalism: public services and citizenship in the information age», Information Infraestructure and Policy, 5.

Teodoro, J., y Arderuu, J. (1999), «El Plan Director para la Societat de la Información en Mataró», III Jomadas de Organización y Nuevas Tecnologias en la Admi. nistración Local, Blanes, 6.8 de octubre de 1999.

Weiner, D., y Vining, A. (1992), Policy Analysis. Concepts and Practice, Prentice Hall, Engelwood Cliffs.

Wuson, E. (1997), «The What, Why, Where and How of National Information Initiatives», en B. KAHIN y E. WLLSON, National Information Infraestructure Initiatives, Cambridge: MIT Press. 